\title{
DE DOS A TRES: CAMBIOS Y NECESIDADES FAMILIARES ANTE EL DESAFÍO DE LA PATERNIDAD
}

\author{
Antonio Urbano Contreras \\ Universidad de Oviedo \\ urbanoantonio@uniovi.es \\ Ma Teresa Iglesias García \\ Universidad de Oviedo \\ Verónica García Díaz \\ Universidad de Oviedo
}

Recepción Artículo: 31 enero 2020

Admisión Evaluación: 2 febrero 2020

Informe Evaluador 1: 4 marzo 2020

Informe Evaluador 2: 4 marzo 2020

Aprobación Publicación: 20 abril 2020

\section{RESUMEN}

La llegada de los hijos al núcleo familiar constituido por la pareja supone una etapa crucial en el ciclo evolutivo familiar, hasta tal punto que algunos autores sitúan aquí el propio inicio de una nueva familia. Además de la importancia que tiene este momento, es evidente que las dinámicas e interacciones familiares previas a la paternidad cambian y, con ello, se generan nuevas necesidades que requieren de apoyo socioeducativo. Concretamente, este trabajo busca analizar las dinámicas de pareja con hijos, para lo que han participado 261 personas (53.6\% mujeres y 46.4\% hombres) mediante un cuestionario de 11 ítems centrados, por un lado, en la toma de decisiones respecto a los hijos y, por otro, en la implicación y dedicación en las tareas de crianza y educación de los hijos. Aunque los resultados generales son positivos, se debe prestar atención a cierta dificultad para llegar a acuerdos con la pareja sobre los hijos y, sobre todo, a la tendencia a que un miembro de la pareja suela ser el que dedique más tiempo a la crianza y cuidado de los hijos, a ayudarles con los deberes y a mantener relación con el centro escolar. El reparto desigual de tales tareas puede estar relacionado con el sentirse desbordado, especialmente en el caso de las mujeres.

Palabras clave: relaciones de pareja; paternidad; crianza de los hijos; familia

\section{ABSTRACT}

From two to three: Family changes and needs facing parenthood. Having children in a household previously constituted by a couple entails a critical phase of the family development cycle, to such an extent that certain authors set in this very moment the beginning of a new family. In addition to the importance of this moment, it is evident that the family dynamics and interactions before the parenthood change and, consequently, new needs requiring socio-educational support arise. Specifically, this research aims to 


\section{DE DOS A TRES: CAMBIOS Y NECESIDADES FAMILIARES ANTE EL DESAFÍ́ DE LA PATERNIDAD}

analyze the dynamics of couples with children; 261 people (53.6\% women and $46.4 \%$ men) completed a questionnaire of 11 items focused, on one side, on the decision-making regarding children and, on the other side, the involvement and commitment on the parenting tasks and the education of their children. Although overall results are positive, attention must also be given to the difficulty in reaching agreements on the children with the partner, and, particularly, who spends more time on upbringing and taking care of their children, helping them with their homework and maintaining the relationship with the school. Unequal household responsibilities may be associated with the fact that women feel more overwhelmed

Keywords: couple relationships; parenthood; child upbringing; family

\section{INTRODUCCIÓN}

Autores como Rodrigo y Palacios (1998) sitúan el momento en el que se pasa de ser pareja a ser pareja y padres como el momento más destacado en la configuración familiar, la cual se verá reorganizada y redefinida si la familia continúa expandiéndose con la llegada de más hijos, pues supondrá la instauración de una nueva dinámica en la que se sumarán a las ya existentes las relaciones entre hermanos. En la misma línea, Hidalgo García y Menéndez Álvarez-Dardet (2009) señalan que la llegada de descendencia es uno de los sucesos vitales más relevantes de la adultez, en gran medida porque implica una gran diversidad de cambios, reajustes y adaptaciones para los nuevos padres, tanto dentro como fuera de la familia. Convertirse en padres pone a prueba los recursos de afrontamiento, pues es un periodo que tiende a caracterizarse como inestable y vulnerable a nivel individual e interpersonal, en el que se generan importantes necesidades de apoyo para afrontar adecuadamente esta significativa experiencia vital.

Convertirse en padres constituye una importante transición que se ve reflejada tanto a nivel personal como familiar. A nivel individual supone un acontecimiento que plantea muchas exigencias e importantes cambios, pues constituirse como padres implica incorporar a la identidad un complejo conjunto de nuevas cogniciones, conductas y emociones, lo que obliga a un proceso de ajuste y adaptación personal que incide directamente en cómo pensar, comportarse y sentirse. Por otra parte, el proceso de convertirse en padres no solo afecta a los adultos y al propio niño, pues el ámbito familiar también se ve modificado ante las nuevas tareas que requiere la llegada de un hijo (nuevas exigencias y horarios, redistribución de papeles, etc.). Por estos motivos, y al igual que ocurre en otras transiciones del ciclo familiar, la transición a la paternidad representa un momento potencialmente propicio para que tengan lugar cambios importantes, los cuales suelen alterar tanto la conducta de los miembros que conforman la familia como las relaciones existentes entre ellos (Hidalgo y Menéndez, 2003).

Estas mismas autoras (2009) destacan que los cambios más sustanciales a nivel interpersonal que conlleva la paternidad son, precisamente, los producidos en la relación de pareja. Concretamente, los cambios más frecuentes que se dan en una relación de pareja cuando se convierten en padres tienen lugar en tres ámbitos, los cuales están relacionados entre sí: 
PSICOLOGÍA Y CRECIMIENTO HUMANO

Tabla 1. Cambios más frecuentes en la relación de pareja con la llegada de los hijos (Adaptación de Hidalgo García y Menéndez Álvarez-Dardet, 2009)

\begin{tabular}{|c|c|c|}
\hline \multicolumn{3}{|c|}{ Principales ámbitos de cambio en una relación de pareia al ser padres } \\
\hline $\begin{array}{c}\text { Actividades } \\
\text { compartidas y hábitos } \\
\text { previos de la pareja }\end{array}$ & $\begin{array}{l}\text { Organización de la } \\
\text { dinámica doméstica }\end{array}$ & $\begin{array}{c}\text { Satisfacción con la relación } \\
\text { conyugal }\end{array}$ \\
\hline $\begin{array}{l}\text { - Disminución del } \\
\text { tiempo dedicado a } \\
\text { rutinas de ocio y } \\
\text { tiempo compartido } \\
\text { - Disminución de la } \\
\text { frecuencia y } \\
\text { satisfacción con las } \\
\text { relaciones sexuales }\end{array}$ & $\begin{array}{l}\text { - Tradicionalización de la } \\
\text { dinámica doméstica (mayor } \\
\text { dedicación de la mujer) } \\
\text { - Incumplimiento de las } \\
\text { expectativas de implicación } \\
\text { y responsabilidad } \\
\text { compartida en la gestión del } \\
\text { hogar y el cuidado de los } \\
\text { hijos }\end{array}$ & $\begin{array}{l}\text { - Cierto decremento en la } \\
\text { intensidad y satisfacción en las } \\
\text { relaciones conyugales } \\
\text { - Las relaciones más positivas } \\
\text { vivirán un declive pero siguen } \\
\text { manteniendo relaciones } \\
\text { positivas y satisfactorias. } \\
\text { - Los problemas ya existentes } \\
\text { antes de ser padres se acentúan }\end{array}$ \\
\hline
\end{tabular}

Además de a nivel de pareja, la llegada de los hijos implica cambios personales y en la esfera de las relaciones sociales (familia extensa, amigos, ámbito laboral). Estos cambios pueden ser positivos, como la intensificación de las relaciones con la familia extensa, en la que se suele encontrar un apoyo considerable, o negativos, como la tendencia a disminuir el contacto y actividades de ocio con amigos (en especial con los que no son padres), la modificación en la implicación en el trabajo e, incluso, dejar de trabajar al convertirse en padres (principalmente en la caso de las madres). En este contexto, y aunque la mayoría de parejas terminan asumiendo de forma satisfactoria y competente su rol de padres, no son pocos los casos en los que esta transición se complica o no se completa adecuadamente, encontrando en estos casos uno de los desencadenantes de muchas rupturas de pareja (Hidalgo García y Menéndez Álvarez-Dardet, 2009).

Al vincular paternidad y otros ámbitos de la pareja, existe cierto consenso en relacionar, por ejemplo, la disminución de la satisfacción sexual, e incluso general, con la llegada de los hijos y el incremento en el número de hijos (Abbott y Brody, 1985; Adams, 1988; Twenge, Campbell y Foster, 2003; Urbano-Contreras, MartínezGonzález e Iglesias-García, 2018), detectándose, principalmente en el caso de las mujeres, una postergación de la satisfacción sexual en favor de la crianza de los hijos. Tal postergación se da sobre todo en los casos en los que no se comparte el cuidado de los hijos, pues aquellas parejas que colaboran de forma igualitaria en dicha tarea reportan mayores índices de calidad en sus relaciones y, concretamente, en su vida sexual (Carlson, Hanson y Fitzroy, 2015).

Ante la influencia que tiene la paternidad en la relación de pareja y, de forma especial, la implicación y el reparto de tareas que conlleva el cuidado de los hijos, esta investigación busca conocer los condicionantes de la relación de pareja asociados a la educación y crianza de los hijos.

\section{MÉTODO}

\section{Participantes}

En este estudio han participado voluntariamente 261 personas que mantenían, en ese momento, una relación de pareja y tenían hijos. El 53.6\% eran mujeres y el $46.4 \%$ hombres. Respecto a su edad, el $18.8 \%$ tenían entre 32 y 38 años, el $22.6 \%$ entre 39 y 45 años, el $26.8 \%$ entre 46 y 52 años, y el $28 \%$ más de 52 años. El $41 \%$ tenían estudios universitarios y el $41,9 \%$ habían mantenido previamente otras relaciones de pareja. 


\section{DE DOS A TRES: CAMBIOS Y NECESIDADES FAMILIARES ANTE EL DESAFÍ́ DE LA PATERNIDAD}

\section{Instrumento}

El instrumento utilizado para recabar la información ha sido un cuestionario dividido en dos partes. En la primera se solicitaba información sobre las variables sexo, edad, nivel de estudios y si habían tenido previamente otras relaciones de pareja. La segunda parte contenía 11 ítems con respuesta en escala Likert de cuatro alternativas, evitando la tendencia a un valor intermedio; Ios tres primeros ítems guardaban relación con la toma de decisiones respecto a los hijos y los otros 8 con la implicación y dedicación en las tareas de crianza y educación de los hijos.

\section{Procedimiento}

Para la selección de la muestra se optó por el método no probabilístico conocido como "Bola de nieve" (snowball sampling) (Goodman, 1961), utilizándose dos procedimientos para la recogida de la información: por un lado, se seleccionó a personas de diferentes edades y niveles culturales que reunían las características esperadas para formar parte de este estudio (tener pareja y tener hijos) y se les entregó un sobre con dos cuestionarios acompañados de una breve carta de presentación e instrucciones de cumplimentación, así como un sobre para cada miembro para devolver su respectivo cuestionario una vez cubierto, y se les pidió que, además de responder al cuestionario, entregaran copias a otras parejas de su entorno y éstas, a su vez a otras, hasta obtener el número necesario; por otro lado, se optó por informatizar el cuestionario, mediante la herramienta de Formularios de Google, para ampliar la muestra dando a conocer primero el cuestionario y el enlace a personas cercanas y pidiéndoles que lo distribuyeran a través de sus redes sociales a otros posibles candidatos que reunieran los requisitos especificados.

\section{Análisis de datos}

Los datos recogidos fueron analizados con el programa SPSS 22.0. En primer lugar, se analizaron las características de la muestra utilizando estadísticos descriptivos (análisis de frecuencias y porcentajes). Las posibles diferencias significativas en función de las variables sexo, nivel de estudios y relaciones de pareja previas se han comprobado mediante el estadístico de contraste $t$ de Student para dos muestras independientes; el tamaño del efecto se ha calculado con el estadístico $d$ de Cohen, considerando que valores de $d$ inferiores a 2 indican un efecto pequeño, entre .2 y .5 un efecto mediano y superiores a .5 un efecto grande. Para comprobar si existían diferencias significativas en función de las variables edad y duración de la pareja se efectuó un análisis de varianza seguido de la aplicación del Test de Scheffé como prueba post-hoc. Finalmente, se calculó la correlación de Pearson entre todas las variables implicadas.

\section{RESULTADOS}

Para facilitar la presentación de los resultados, estos se dividen en dos bloques: 1) Toma de decisiones respecto a las cuestiones relaciones con los hijos y 2) Implicación y dedicación en las tareas de crianza y educación de los hijos. Se comienza el análisis presentando la distribución de las respuestas a los ítems y las diferencias estadísticamente significativas observadas en base a las variables de clasificación establecidas para, posteriormente, describir las correlaciones existentes entre los ítems de ambas dimensiones.

Toma de decisiones respecto a los hijos

Esta dimensión, reflejada en la Tabla 2, muestra que las parejas suelen tomar conjuntamente las decisiones que afectan a los hijos y no suele existir una predilección por decidir de forma unilateral las cuestiones relacionadas con la educación de estos, pero parece darse en ocasiones cierta dificultad para llegar a acuerdos con la pareja sobre los hijos. 
PSICOLOGÍA Y CRECIMIENTO HUMANO

Tabla 2. Toma de decisiones respecto a los hijos

\begin{tabular}{lcccccc}
\hline \multicolumn{1}{c}{ Ítems } & Nunca & $\begin{array}{c}\text { A } \\
\text { veces }\end{array}$ & $\begin{array}{c}\text { Casi } \\
\text { siempre }\end{array}$ & Siempre & M & Sx \\
\hline $\begin{array}{l}\text { 1. Mi pareja y yo tomamos } \\
\text { conjuntamente las decisiones que } \\
\text { afectan a los hijos }\end{array}$ & $3.3 \%$ & $8.2 \%$ & $32.2 \%$ & $56.3 \%$ & 3.42 & .78 \\
\hline $\begin{array}{l}\text { 2. Prefiero ser yo quien tome las } \\
\text { decisiones en cuanto a la } \\
\text { educación de los hijos }\end{array}$ & $56.6 \%$ & $24.7 \%$ & $12.3 \%$ & $6.4 \%$ & 1.69 & .92 \\
\hline $\begin{array}{l}\text { 3. Es fácil llegar a acuerdos con mi } \\
\text { pareja sobre los hijos }\end{array}$ & $2.9 \%$ & $19.7 \%$ & $34.7 \%$ & $42.7 \%$ & 3.17 & .85 \\
\hline
\end{tabular}

Respecto al contraste entre grupos, el ítem 3 es el único que presenta diferencias significativas ( $p=.000, d=-$ .47), en el que los hombres, las personas no universitarias $(\mathrm{p}=.015, \mathrm{~d}=.32)$ y las que no han tenido alguna relación con anterioridad ( $p=.031$, $d=.29$ ) parecen percibir que es más fácil llegar a acuerdos sobre los hijos con sus parejas con tamaños del efecto entre medianos y grandes. En cuanto a las diferencias por edad y duración de la relación, no se han encontrado diferencias significativas.

Implicación y dedicación en las tareas de crianza y educación de los hijos

Respecto a esta dimensión (Tabla 3), destaca positivamente el alto porcentaje de parejas que hablan diariamente sobre aspectos que incumben a los hijos. Sin embargo, un aspecto no tan positivo es observar la variabilidad existente a la hora de considerar que un único miembro de la pareja suele ser el que dedica tiempo a las tareas de crianza y cuidado de los hijos, ayudarles con los deberes y mantener relación con el centro escolar, lo que puede estar vinculado con el porcentaje de personas que indican que "A veces" o "Casi siempre" se sienten desbordadas por el reparto desigual de tales tareas, cuestiones que se entiende y explican mejor al analizar las diferencias por sexos. Además, destaca el porcentaje de personas que encuentra en el comportamiento de los hijos una fuente de conflictos entre la pareja. 
DE DOS A TRES: CAMBIOS Y NECESIDADES FAMILIARES ANTE EL DESAFÍ́ DE LA PATERNIDAD

Tabla 3. Implicación y dedicación en las tareas de crianza y educación de los hijos

\begin{tabular}{|c|c|c|c|c|c|c|}
\hline Ítems & Nunca & $\begin{array}{c}\mathrm{A} \\
\text { veces }\end{array}$ & $\begin{array}{c}\text { Casi } \\
\text { siempre }\end{array}$ & Siempre & $\mathrm{M}$ & $\mathrm{Sx}$ \\
\hline $\begin{array}{l}\text { 4. Hablamos diariamente sobre } \\
\text { aspectos que incumben a los hijos }\end{array}$ & $1.3 \%$ & $14.5 \%$ & $37.2 \%$ & $47 \%$ & 3.30 & .76 \\
\hline $\begin{array}{l}\text { 5. Soy yo quien suele dedicar } \\
\text { tiempo a las tareas de crianza y } \\
\text { cuidado de los hijos }\end{array}$ & $26.5 \%$ & $32.3 \%$ & $24.8 \%$ & $16.4 \%$ & 2.31 & 1.04 \\
\hline $\begin{array}{l}\text { 6. Suelo ser yo quien dedica } \\
\text { tiempo a ayudar a los hijos con los } \\
\text { deberes }\end{array}$ & $25 \%$ & $33.2 \%$ & $24.1 \%$ & $17.7 \%$ & 2.35 & 1.04 \\
\hline $\begin{array}{l}\text { 7. Soy yo quien suele acudir y } \\
\text { mantener relación con el centro } \\
\text { escolar de los hijos }\end{array}$ & $25.8 \%$ & $27.6 \%$ & $26.7 \%$ & $19.9 \%$ & 2.41 & 1.08 \\
\hline $\begin{array}{l}\text { 8. El reparto desigual de las tareas } \\
\text { de cuidado y educación de los } \\
\text { hijos es una fuente de conflicto } \\
\text { entre mi pareja y yo }\end{array}$ & $55 \%$ & $28.6 \%$ & $9.1 \%$ & $7.4 \%$ & 1.69 & .92 \\
\hline $\begin{array}{l}\text { 9. El comportamiento de los hijos } \\
\text { genera conflictos entre mi pareja } \\
\text { y yo }\end{array}$ & $32.3 \%$ & $38.8 \%$ & $19.8 \%$ & $9.1 \%$ & 2.06 & .94 \\
\hline $\begin{array}{l}\text { 10. En el día a día me siento } \\
\text { desbordado por el trabajo y la } \\
\text { implicación que conlleva tener } \\
\text { hijos }\end{array}$ & $41.6 \%$ & $37.7 \%$ & $16.5 \%$ & $4.3 \%$ & 1.84 & .85 \\
\hline $\begin{array}{l}\text { 11. Mi pareja y yo necesitamos } \\
\text { ayuda para saber cómo atender y } \\
\text { educar a los hijos }\end{array}$ & $66.4 \%$ & $29.3 \%$ & $2.6 \%$ & $1.7 \%$ & 1.40 & .63 \\
\hline
\end{tabular}

Considerando el contraste de grupos, los resultados muestran diferencias significativas en cuanto al sexo en los ítems $6(p=.000, d=.65), 7(p=.000, d=.78)$ y $10(p=.037, d=.28)$, siendo las mujeres las que dedican más tiempo a ayudar a los hijos con los deberes, las que suelen mantener relación con el centro escolar y las que en mayor medida se sienten desbordadas por el trabajo y la implicación que conlleva tener hijos, con tamaños del efecto entre medianos y grandes.

Por otro lado, el nivel de estudios únicamente presenta tales diferencias en el ítem $5(p=.000, d=.50)$, siendo la población no universitaria la que presenta mayores diferencias entre los miembros de la pareja a la hora de repartir las tareas de crianza y cuidado de los hijos, con un tamaño del efecto grande. Por último, aquellas personas que han tenido alguna relación con anterioridad presentan un reparto más desigual en cuanto a las tareas comentadas (ítem $5, \mathrm{p}=.002, \mathrm{~d}=.37$ ) y superior ante la necesidad de ayuda para saber cómo atender y educar a Ios hijos (ítem 11, p=.047, d=-.31), en ambos casos con tamaños del efecto medianos.

Por último, para analizar las diferencias significativas por edad y por el tiempo de duración de la relación, se opta por presentar los resultados del análisis de la varianza con un factor en la Tabla 4: 
Tabla 4. Diferencias significativas en la implicación y dedicación en las tareas de crianza y educación de los hijos en base a la edad y el tiempo de duración de la relación

\begin{tabular}{|c|c|c|c|}
\hline Ítems & Factor & $\mathrm{p}$ & $\begin{array}{l}\text { Interpretación y diferencia por grupos } \\
\text { (Scheffe) }\end{array}$ \\
\hline $\begin{array}{c}4 \text { (Hablar sobre los } \\
\text { hijos) }\end{array}$ & Edad & .007 & $\begin{array}{l}\text { Diferencias significativas entre el tercer } \\
\text { intervalo y el cuarto }(.016) \text {. A menor edad } \\
\text { mayor comunicación. }\end{array}$ \\
\hline \multirow{2}{*}{$\begin{array}{l}5 \text { (Crianza y } \\
\text { cuidado) }\end{array}$} & Edad & .000 & $\begin{array}{l}\text { No hay diferencias significativas entre } \\
\text { grupos. }\end{array}$ \\
\hline & Duración & .000 & $\begin{array}{l}\text { No hay diferencias significativas entre } \\
\text { grupos. }\end{array}$ \\
\hline 6 (Deberes) & Edad & .044 & $\begin{array}{l}\text { No hay diferencias significativas entre } \\
\text { grupos. }\end{array}$ \\
\hline \multirow{2}{*}{$\begin{array}{l}10 \text { (Sentirse } \\
\text { desbordado) }\end{array}$} & Edad & .003 & $\begin{array}{l}\text { Diferencias significativas entre el tercer } \\
\text { intervalo y el cuarto }(.010) \text {. A menor edad } \\
\text { mayor sentimiento }\end{array}$ \\
\hline & Duración & .003 & $\begin{array}{l}\text { Diferencias significativas entre el cuarto } \\
\text { intervalo y el quinto }(.044) \text {. A menor } \\
\text { duración mayor sentimiento. }\end{array}$ \\
\hline \multirow{2}{*}{$\begin{array}{c}11 \text { (Necesitamos } \\
\text { ayuda) }\end{array}$} & Edad & .002 & $\begin{array}{l}\text { Diferencias significativas entre el tercer } \\
\text { intervalo y el cuarto }(.020) \text {. A menor edad } \\
\text { mayor necesidad de ayuda }\end{array}$ \\
\hline & Duración & .008 & $\begin{array}{l}\text { No hay diferencias significativas entre } \\
\text { grupos. }\end{array}$ \\
\hline
\end{tabular}

\section{Análisis correlacional}

En cuanto a las correlaciones analizadas (Tabla 5), destaca en primer lugar las correlaciones positivas entre la toma de decisiones compartida en las cuestiones que afectan a los hijos (ítem 1) y aspectos como la facilidad para llegar a acuerdos (ítem 3) y hablar diariamente sobre cuestiones que incumben a los hijos (ítem 4), mientras que se dan correlaciones negativas atendiendo a la preferencia de tomar decisiones individualmente (ítem 2) o la existencia de conflictos de pareja por el comportamiento de los hijos (ítem 9) y el reparto desigual de las tareas de cuidado y educación de estos (ítem 8).

En aquellos casos en los que se prefiere tomar las decisiones referentes a los hijos de forma más individual (ítem 2), aparecen correlaciones positivas en la asunción mayoritaria de tareas como la crianza (ítem 5), la ayuda con los deberes (ítem 6) y el contacto con el centro escolar de los hijos (ítem 7), vinculándose a una mayor conflictividad entre la pareja por el reparto desigual de tareas (ítem 8) y con una menor facilidad para llegar a acuerdos sobre los hijos (ítem 3).

Por último, destacan las correlaciones positivas moderadas que se dan entre la percepción de necesitar ayuda para atender y educar a los hijos (ítem 11) y aquellas parejas que manifiestan contar con conflictos de pareja como consecuencia del reparto desigual de tareas (ítem 8) y el comportamiento de los hijos (ítem 9), así como aquellas que se sienten desbordadas por el trabajo y la implicación que conlleva tener hijos (ítem 10). Además, estas tres situaciones también presentan correlaciones positivas moderadas entre sí. 


\section{DE DOS A TRES: CAMBIOS Y NECESIDADES FAMILIARES ANTE EL DESAFÍO DE LA PATERNIDAD}

Tabla 5. Correlaciones bivariadas referentes al bloque sobre educación y crianza de los hijos

\begin{tabular}{|c|c|c|c|c|c|c|c|c|c|c|}
\hline & 1 & 2 & 3 & 4 & 5 & 6 & 7 & 8 & 9 & 10 \\
\hline 1.DecidirHiJuntos & 1 & & & & & & & & & \\
\hline $\begin{array}{l}\text { 2.DecidoYoEducaci } \\
\text { ón }\end{array}$ & $\begin{array}{c}- \\
.36^{*}\end{array}$ & 1 & & & & & & & & \\
\hline 3.AcuerdoHiFácil & $.36^{*}$ & $.26^{*}$ & 1 & & & & & & & \\
\hline 4.HablamosHi & $.44^{*}$ & $\begin{array}{c}- \\
.17^{*}\end{array}$ & $.40^{*}$ & 1 & & & & & & \\
\hline 5.CrianzaYoHi & -.10 & $.43^{*}$ & $.22^{*}$ & $\begin{array}{c}- \\
.08\end{array}$ & 1 & & & & & \\
\hline 6.DeberesYoHi & -.12 & $.41^{*}$ & $.22^{*}$ & .06 & $.73^{*}$ & 1 & & & & \\
\hline 7.EscolarYoHi & -.12 & $.40^{*}$ & $.17^{*}$ & $\begin{array}{c}- \\
.02\end{array}$ & $.70^{*}$ & $.73^{*}$ & 1 & & & \\
\hline 8.RepartoDesigual & $.20^{-}$ & $.26^{*}$ & $.22^{*}$ & $\begin{array}{c}- \\
.07\end{array}$ & $.14^{*}$ & $.18^{*}$ & $.19^{*}$ & 1 & & \\
\hline $\begin{array}{l}\text { 9.ProblemHiCompo } \\
\text { rta }\end{array}$ & $.22^{-}$ & .11 & $.34^{*}$ & $\begin{array}{c}- \\
.13\end{array}$ & .08 & $.13^{*}$ & $.14^{*}$ & $.37^{*}$ & 1 & \\
\hline 10.DesbordaHi & $\begin{array}{c}- \\
.18^{*}\end{array}$ & .09 & $.18^{*}$ & $\begin{array}{c}- \\
.03\end{array}$ & $.18^{*}$ & $.20^{*}$ & $.19^{*}$ & .30 & $.43^{*}$ & 1 \\
\hline 11.AyudaHi & $-15^{*}$ & .11 & -.11 & $\begin{array}{c}- \\
.08\end{array}$ & .10 & .10 & .12 & $.32^{*}$ & $.33^{*}$ & $.38^{*}$ \\
\hline
\end{tabular}

$\mathrm{Hi}=\mathrm{Hijo} / * \mathrm{p}<.05 ; * * \mathrm{p}<.0$

\section{DISCUSIÓN Y CONCLUSIONES}

En función de los resultados expuestos, destaca que parece existir cierto consenso entre las parejas a la hora de tomar decisiones y cierta predisposición a querer tomar tales decisiones de forma conjunta, aunque se ha de trabajar para incrementar la facilidad para llegar a acuerdos entre las parejas en temas que incumben a los hijos.

En contraposición con este aparente acuerdo en la toma de decisiones sobre los hijos, en el plano concreto de la dedicación e implicación en las tareas que conlleva la crianza y educación de los hijos, se evidencia cierta desigualdad de roles, en perjuicio para las mujeres, en aspectos como la ayuda con los deberes, el mantenimiento de relaciones con el centro educativo o la dedicación de tiempo a las tareas de atención básica hacia los hijos, lo que puede estar asociado a que ellas también se sientan más desbordadas por el trabajo e implicación que conlleva tener hijos. Esta cuestión evidencia una doble necesidad, trabajar en la línea de lograr una igualdad efectiva en el reparto de tareas y funciones dentro del hogar y, por otro lado, favorecer la incorporación activa de la figura paterna en otras esferas como son las relaciones con el centro escolar de los hijos. 
Esta desigualdad de género, y la sobrecarga que todavía parece soportar la mujer, puede estar muy relacionada con los elementos vinculados al ámbito de la satisfacción con la relación de pareja, en el que la mujer suele ser la que ve mermada en mayor medida la satisfacción cuando la pareja cuenta con hijos (Medina, Lederhos y Lillis, 2009; Rholes, Simpson, Campbell y Grich, 2001; Urbano-Contreras, Iglesias-García y Martínez-González, 2019).

Además, tal y como afirma Torres (2004), en la división de las tareas domésticas y el cuidado de los hijos podría estar el origen de muchos conflictos entre la pareja. Esta autora, tras analizar la relación entre ambas cuestiones en el contexto portugués, llegó a la conclusión que el 37\% de las personas indicaban que, algunas 0 muchas veces, se generaban conflictos por el reparto de las tareas domésticas y el 28\% los tenía por cuestiones relacionadas con el cuidado de los hijos.

Respecto a este tema, como recogen Garrido Garduño, Reyes Luna, Ortega Silva y Torres Velásquez (2007) constituirse simplemente como pareja es relativamente fácil, pero otra cuestión es asumir las exigencias y responsabilidades que conlleva el día a día, en el que es imprescindible mantener el diálogo y la compresión, y en la el que no siempre tiene lugar una tarea compartida de la crianza y educación de los hijos.

Para intentar facilitar el desarrollo de un rol parental compartido y positivo para ambas figuras parentales, los datos parecen indicar que es necesario, por un lado, compartir las decisiones que afectan a los hijos y hablar diariamente sobre las cuestiones que les incumben, pues esto facilita llegar a acuerdos entre la pareja y, por otro, evitar el reparto desigual de las tareas que conlleva el cuidado y educación de los hijos y la toma de decisiones unilateral, pues parecen ser dos cuestiones que favorecen no llegar a acuerdos con facilidad y la existencia de conflictos entre las parejas motivados, por ejemplo, por el comportamiento de los hijos.

Además, el comportamiento de los hijos como detonante del conflicto entre la pareja merece una especial atención, ya que supone una alteración de la vida diaria que implica a más de un subsistema familiar, lo que complica su abordaje y las posibilidades de acabar percibiendo una respuesta positiva al conflicto por parte de la pareja, cuestión que repercutirá, además, en la calidad percibida de la propia relación (Greeff y De Bruyne, 2000; Paleari, Regalia y Fincham, 2010). Para reducir los conflictos en esta línea, parece fundamental, en primer lugar, ofrecer a las parejas las competencias necesarias para saber establecer acuerdos, pero también es importante reducir la percepción de sentirse desbordado por el trabajo e implicación que conlleva tener hijos, para lo que pueden resultar de gran utilidad las técnicas de relajación y autorregulación emocional, así como favorecer momentos y espacios en los que uno pueda contar con tiempo para dedicarlo a uno mismo.

\section{REFERENCIAS BIBLIOGRÁFICAS}

Abbott, D. y Brody, G. (1985). The relation of child age, gender, and number of children to the marital adjustment of wives. Journal of Marriage and the Family, 47, 77-84.

Adams, W. J. (1988). Sexuality and happiness ratings of husbands and wives in relation to first and second pregnancies. Journal of Family Psychology, 2(1), 67-81.

Carlson, D. L., Hanson, S. y Fitzroy, A. (2015). The Division of Childcare, Sexual Intimacy, and Relationship Quality in Couples. Sociology Faculty Publications, Paper 4.

Garrido Garduño, A., Reyes Luna, A. G., Ortega Silva, P. y Torres Velásquez, L. E. (2007). La vida en pareja: un asunto a negociar. Enseñanza e investigación en Psicología, 12(2), 385-396.

Goodman, L. A. (1961). Snowball Sampling. Annals of Mathematical Statistics, 32, 148-170.

Greeff, A. P. y De Bruyne, T. (2000). Conflict management style and marital satisfaction. Journal of Sex and Marital Therapy, 26(4), 321-334.

Hidalgo, M. V. y Menéndez, S. (2003). La pareja ante la llegada de los hijos e hijas. Evolución de la relación conyugal durante el proceso de convertirse en padre y madre. Infancia y Aprendizaje, 26(4), 469-483. doi: 10.1174/021037003322553851

Hidalgo García, M. V. y Menéndez Álvarez-Dardet, S. (2009). Apoyo a las familias durante el proceso de transición a la maternidad y la paternidad. Familia: Revista de Ciencias y Orientación Familiar, 38, 133-152. 
Medina, A. M., Lederhos, C. L. y Lillis, T. A. (2009). Sleep disruption and decline in marital satisfaction across the transition to parenthood. Families, Systems and Health, 27(2), 153-160.

Paleari, F. G., Regalia, C. y Fincham, F. D. (2010). Forgiveness and Conflict Resolution in Close Relationships: Within and Cross Partner Effects. Universitas Psychologica, 9(1), 35-56.

Rholes, W. S., Simpson, J. A., Campbell, L. y Grich, J. (2001). Adult attachment and the transition to parenthood. Journal of personality and social psychology, 81(3), 421-435. doi: 10.1037/0022-3514.84.6.1172

Rodrigo, M. J. y Palacios, J. (Coords). (1998). Familia y desarrollo humano. Madrid: Alianza Editorial S.A.

Torres, A. C. (2004). Vida Conjugal e Trabalho. Oeiras, Portugal: Celta Editora.

Twenge, J. M., Campbell, W. K. y Foster, C. A. (2003). Parenthood and marital satisfaction: A meta-analytic review. Journal of Marriage and the Family, 65, 574-583.

Urbano-Contreras, A., Iglesias-García, M. T. y Martínez-González, R. A. (2019). Satisfacción general y sexual con la relación de pareja en función del género. Revista Española de Investigaciones Sociológicas, 165, 143-158.

Urbano-Contreras, A., Martínez-González, R. A. y Iglesias-García, M. T. (2018). Parenthood as a determining factor of satisfaction in couple relationships. Journal of Child and Family Studies, 27(5), 1492-1501. 